\title{
Medium-term outcomes after laparoscopic revision of laparoscopic Kasai portoenterostomy in patients with biliary atresia
}

Yi Ji

Sichuan University West China Hospital https://orcid.org/0000-0002-9289-9660

\section{Xuepeng Zhang}

Sichuan University West China Hospital

Siyuan Chen

Sichuan University West China Hospital

Yanan Li

Sichuan University West China Hospital

\section{Kaiying Yang}

Sichuan University West China Hospital

Jiangyuan Zhou

Sichuan University West China Hospital

Zhicheng Xu ( $\nabla$ xzcxewk@163.com)

West China Hospital of Sichuan University

\section{Research}

Keywords: Biliary atresia, Laparoscopic Kasai portoenterostomy, Revision, Outcomes

Posted Date: September 3rd, 2020

DOl: https://doi.org/10.21203/rs.3.rs-70828/v1

License: (c) (i) This work is licensed under a Creative Commons Attribution 4.0 International License.

Read Full License

Version of Record: A version of this preprint was published at Orphanet Journal of Rare Diseases on April 30th, 2021. See the published version at https://doi.org/10.1186/s13023-021-01835-z. 


\section{Abstract \\ Objective}

To determine whether revision laparoscopic Kasai portoenterostomy (RLKPE) is a viable treatment option for patients with biliary atresia (BA) who underwent initially successful laparoscopic Kasai portoenterostomy (ILKPE).

\section{Methods}

The medical records of 312 patients with nonsyndromic BA who underwent ILKPE between May 2009 and May 2017 were retrospectively reviewed. The patients were divided into three groups according to their outcomes after ILKPE: group A: 25 patients who underwent RLKPE; group B: 203 patients who underwent ILKPE and required no further surgical intervention; and group C: 84 patients with failed ILKPE who either died or required liver transplantation for survival. The 3-year and 5-year survival with native liver (SNL) rates were compared between groups $A$ and $B$ and between groups $A$ and $C$. Among the 25 patients in group $A$, the perioperative data of RLKPE were compared with those of ILKPE.

\section{Results}

Of the 312 patients who underwent ILKPE, 228 reached the normal bilirubin concentration range within 6 months postoperatively. Among them, 25 patients with a sudden cessation of bile flow underwent RLKPE. Adequate biliary drainage evidenced by normalized conjugated bilirubin levels was achieved in $80 \%$ of the patients who underwent RLKPE. The perioperative variables, including operative time, blood loss, rate of conversion to open surgery and complications of RLKPE, were not significantly different between RLKPE and ILKPE. The 3-year and 5-year SNL rates in patients after RLKPE were $64.0 \%$ and $52.0 \%$, respectively, which were not significantly different from the corresponding $86.2 \%$ and $73.9 \%$ in patients after unrevised ILKPE $(P>0.05)$ but were significantly better than the corresponding values of group $\mathrm{C}(P<0.01)$.

\section{Conclusion}

Our data demonstrated that with appropriate patient selection, RPLKE can be a viable and effective treatment opinion in patients who experience sudden cessation of bile drainage after ILKPE. RPLKE can delay the need for liver transplantation, yielding encouraging medium-term patient outcomes.

\section{Introduction}

Biliary atresia (BA) is a progressive fibrous occlusive disease that, if not treated promptly and effectively, may lead to cirrhosis or end-stage liver disease [1]. Although liver transplantation (LT) significantly 
improves the prognosis of patients with BA, Kasai portoenterostomy is still regarded as the standard initial option due to the technical complexity associated with liver transplantation in young children and the shortage of qualified donors [2, 3]. In 2002, Esteves [4] developed the laparoscopic Kasai portoenterostomy (LKPE) procedure, which created a new era of minimally invasive surgery for BA. Although a learning curve of LKPE is present, some satisfactory results have been achieved, in which the 3-year and 5-year survival with native liver (SNL) rates after LKPE were not inferior, or even superior, to those after open Kasai portoenterostomy (OKPE) [5-10]. Unfortunately, LKPE is not effective in every patient with $B A$, as is the case for OKPE. In patients who experienced sudden cessation of bile drainage after initially successful LKPE (ILKPE), the necessity and indications for revision LKPE (RLKPE) have not been previously reported. Herein, we review and analyze our experiences with RLKPE in patients with BA.

\section{Materials And Methods}

\subsection{Design and study population}

This study was approved by the Ethical Committee of the West China Hospital of Sichuan University. The patients' parents or guardians gave written informed consent. The diagnosis of BA was based on a combination of ultrasonic findings, intraoperative cholangiography findings, and histological examinations of the hepatic parenchyma and extrahepatic bile ducts. Patients with BA splenic malformation, severe cardiac anomalies, or other significant comorbidities that would influence the postoperative course were excluded from the study. The 312 patients with nonsyndromic BA who underwent LKPE from May 2009 to May 2017 were enrolled in this study. They were divided into 3 cohorts based on their outcome after ILKPE: (1) the 25 patients in group A underwent RLKPE; (2) the 203 patients in group B underwent ILKPE and required no further surgical intervention; and (3) the 84 patients in group C experienced failed ILKPE and either died or required liver transplantation for survival. The indications for revision were the sudden cessation of bile drainage after ILKPE and no improvement after 2 weeks of antibiotic treatment. The 3-year and 5-year SNL rates were compared between groups $A$ and $B$ and between groups $A$ and $C$. Among the patients in group $A$, the perioperative data, rates of clearance of jaundice (CJ) and cholangitis of RLKPE were compared with those of ILKPE.

\subsection{Operative technique}

\subsubsection{The LKPE procedure}

The LKPE procedure was similar to that described in a previously published paper [5-7]. Succinctly, a percutaneous suture was used to snare the round and falciform ligament and to retract the liver. The atretic gallbladder and cystic duct are dissected free from the gallbladder fossa. The portal plate over the bifurcation of the main portal vein could be found along the common hepatic duct. Once the base of the fibrous cone was reached, the fibrous cord was transected with laparoscopic scissors. The resection level of the fibrous cone was dependent on the presence of abundant bile-like juice over the fibrous stump. Bleeding from the fibrous remains of the portal plate was controlled by direct compression with gauze pads. The ligament of Treitz was identified, and the jejunum $15 \mathrm{~cm}$ distal to the ligament was exteriorized 
through the umbilical port site. A 25-40 cm Roux-en-Y limb was fashioned. The Roux loop was delivered to the hilum through a retrocolic path. One layer of end-to-side portoenterostomy (diameter of the anastomosis 1.0-1.5 cm) was performed with interrupted 5-0 absorbable sutures. A drain was inserted into the foramen of Winslow.

\subsubsection{The RLKPE procedure}

The trocar placement and pneumoperitoneum pressure settings were the same as those in LKPE, as mentioned above. The other steps of RLPKE were as follows: (1) removing the greater omentum adhered to the porta hepatis (Fig 1 a); (2) partly dismantling the anastomosed Roux limb adjacent to the porta hepatis (Fig 1 b); (3) resecting the granulation tissue; (4) dissecting the hilar fibrous stump layer by layer until abundant bile-like juice was observed (Fig $1 \mathrm{c}$, d); and (5) carrying out end-to-end portoenterostomy after trimming the tail of the Roux limb.

\subsection{The management algorithm after surgery}

Postoperatively, the BA patients in the 3 groups received the same medical treatment protocol. Antibiotic therapy was continued intravenously for 25-30 days in the hospital. Methylprednisolone was administered intravenously 5 days postoperatively at a dose of $5 \mathrm{mg} / \mathrm{kg} /$ day initially and was reduced by $1 \mathrm{mg} / \mathrm{kg} /$ day every 3 days for 2 weeks or longer until a normal total bilirubin value was reached.

Sulfamethoxazole and cephalosporin antibiotics were orally administered alternately weekly until 1 year of age. Ursodeoxycholic acid and hepatoprotective tablets were used until 3 years of age.

\subsection{Definitions}

The operative time (ORT) was calculated as the length of time between the skin incision and closure. Any perioperative complications (APOC) was defined as a complication that occurred during the perioperative period, including wound infection, umbilical hernia, intestinal anastomotic fistula, and adhesive intestinal obstruction. CJ was defined as a total bilirubin level $<1.2 \mathrm{mg} / \mathrm{dL}$ within 6 months postoperatively. Cholangitis was defined as an elevated serum bilirubin $(>2.5 \mathrm{mg} / \mathrm{dL})$, leukocytosis with left shift, and normal to acholic stools in a febrile patient $\left(>38.0^{\circ} \mathrm{C}\right)$. Postoperative outcomes were followed through outpatient clinic evaluations and were excluded if the patients underwent liver transplantation or died.

\subsection{Statistics}

The software applied for statistical calculations was SPSS 22.0 for Windows 10.0 (SPSS Inc., Chicago, IL, USA). The demographic data of BA patients were compared using one-way analysis of variance and the chi-squared test. The difference in SNL rate among the patients was analyzed by the Kaplan-Meier method with endpoints of death or liver transplantation and compared using the log-rank test. A $P$ value $<0.05$ was considered statistically significant.

\section{Results}




\subsection{Demographic and clinical characteristics of all patients in the three groups}

From May 2009 to May 2019, 312 patients with nonsyndromic BA underwent ILKPE. The demographic and clinical characteristics of all patients in the three groups are presented in Table 1. The median age at operation was $82.8 \pm 19.4$ days, with a male proportion of $32.1 \%$. The proportions of types I, II, and III and cystic BA were $1.6 \%, 4.8 \%, 82.4 \%$ and $11.2 \%$, respectively. The median follow-up time was $67.2 \pm$ 25.5 months. The rates of $\mathrm{CJ}$ and cholangitis after ILKPE were $73.1 \%$ and $58.9 \%$, respectively. The 3-year and 5-year SNL rates were $62.6 \%$ and $54.1 \%$, respectively.

Table 1

Demographic and clinical characteristics of all patients who underwent ILKPE

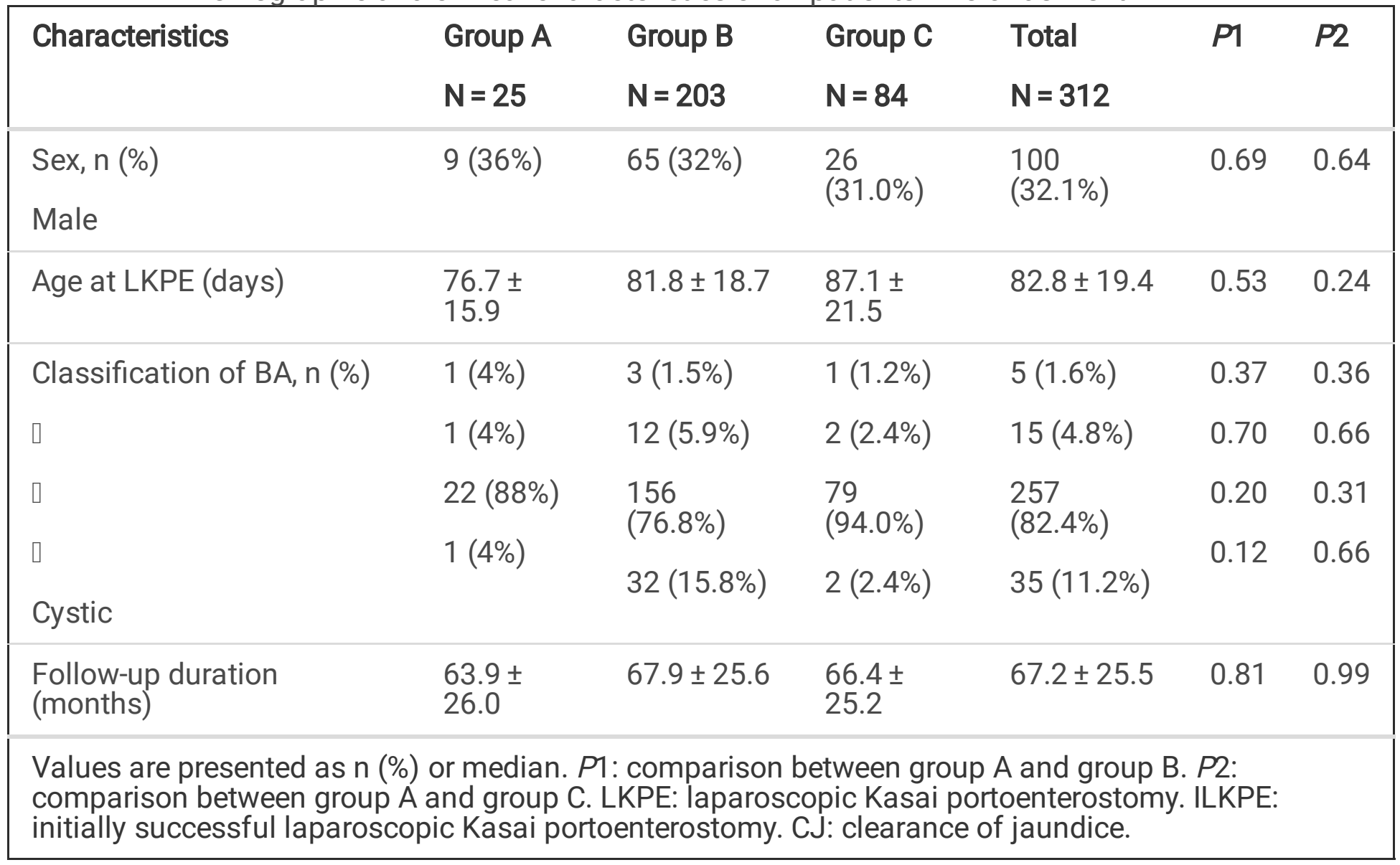

\subsection{Comparison Between Group A And Group B}

In group A, 25 patients underwent RLKPE. The male proportion, age and follow-up time were not significantly different between groups A and B. The 3-year and 5-year SNL rates were $64.0 \%$ and $52.0 \%$ in group $A$ and were not significantly different from the $86.2 \%$ and $73.9 \%$ in group $B(P>0.05)$ (Fig. 2).

\subsection{Comparison Between Group A And Group C}


The male proportion, age and follow-up time were not significantly different between group A and group C. The 3-year and 5-year SNL rates were $64.0 \%$ and $52.0 \%$ in group $A$ and were significantly better than those in group $C(P<0.01)$ (Fig. 3).

\subsection{Comparison of perioperative variables and rates of $\mathrm{CJ}$ and cholangitis between ILKPE and RLKPE in 25 patients}

The demographic and clinical characteristics of 25 patients who underwent both ILKPE and RLKPE are presented in Table 2. The ages at ILKPE and RLKPR were $76.7 \pm 15.9$ days and $159.8 \pm 50.4$ days, respectively. The time interval from suspension of bile drainage to revision was $21.9 \pm 8.0$ days. The ORT, blood loss, conversion rate and incidence of AOPC during ILKPE were not significantly different from those during RLKPE. The rates of CJ and cholangitis after ILKPE were $100 \%$ and $64.0 \%$ and were significantly different from the $80.0 \%$ and $28.0 \%$ after RLKPE $(P<0.01)$.

Table 2

Demographic and clinical characteristics of the 25 patients who underwent both ILKPE and RLKPE

\begin{tabular}{|llll|}
\hline Characteristic & ILKPE & RLKPE & $P$ \\
\hline Age at LKPE, (days) & $76.7 \pm 15.9$ & $159.8 \pm 50.4$ & 0.00 \\
\hline ORT, (min) & $241.1 \pm 32.4$ & $179.6 \pm 29.5$ & 0.75 \\
\hline Conversion rate, $\mathrm{n}(\%)$ & $0(0.0)$ & $1(4.0)$ & 0.31 \\
\hline Median blood loss, (ml) & $30.4 \pm 19.2$ & $23.0 \pm 11.0$ & 0.07 \\
\hline APOC, $\mathrm{n}(\%)$ & $2(8.0)$ & $1(4.0)$ & 0.55 \\
\hline CJ, $\mathrm{n}(\%)$ & $25(100.0)$ & $20(80.0)$ & 0.00 \\
\hline Cholangitis, $\mathrm{n}(\%)$ & $16(64.0)$ & $7(28.0)$ & 0.00 \\
\hline $\begin{array}{l}\text { Values are presented as n (\%) or median. LKPE: laparoscopic Kasai portoenterostomy. ILKPE: initially } \\
\text { successful laparoscopic Kasai portoenterostomy. CJ: clearance of jaundice. ORT: operative time. } \\
\text { APOC: any perioperative complication }\end{array}$ & & \\
\hline
\end{tabular}

\section{Discussion}

Experiences with revision for BA patients with recurrent jaundice after initial OKPE or ILKPE have rarely been reported. In two studies, CJ rates of $60 \%$ [11] and 83.3\% [12] were obtained after revision of OKPE for patients with initially successful OKPE. Naruhiko et al. showed good results of RLKPE for patients with recurrent jaundice after initial OKPE. The authors found that 10/12 patients had normal bilirubin concentrations after RLKPE [13]. In our study, 20/25 (80\%) patients with sudden cessation of bile drainage achieved normal bilirubin concentrations after RLKPE. In addition, the 3-year and 5-year SNL rates of patients who underwent RLKPE were comparable to those of patients after unrevised ILKPE and were significantly better than those of patients after failed ILKPE. These satisfactory results were attributed to a rigorous selection of candidates with poor jaundice reduction after the initial Kasai 
operation. If the indications for revision were relaxed, the postoperative results would be quite different. In a survey from the Japanese Biliary Atresia Registry, the revision rate was $21 \%$ and the $\mathrm{CJ}$ rate was $35 \%$ among 2630 BA patients after the failed initial OKPE during 1989-2011 [10].

The indications for RLKPE in our center were bile drainage that stopped abruptly after ILKPE and no improvement after 2 weeks of antibiotic treatment. However, a consensus has never been reached for the optimal timing of revision worldwide. Some reports have shown that revision could be effective for patients in whom jaundice suddenly recurred after a favorable initial reduction, irrespective of the time since the initial surgery $[14,15]$. However, Shirota suggested that the revision should be performed as soon as irreversible jaundice is recognized [12]. In our study, the time interval from the cessation of bile drainage to revision was $21.9 \pm 8.0$ days, which should be shortened to avoid further liver damage. RLKPE is not recommended for patients with bile drainage failure for more than 60 days or patients with severe ascites or aggravated liver function. For patients over 1 year old with sudden bile drainage failure, antibiotics should be administered first, and liver transplantation is recommended if conservative treatment is ineffective.

Technically, RLKPE is relatively easy to perform, partly due to the omission of the Roux limb anastomosis, which is a time-consuming step in ILKPE. Under the magnified and clear view of the laparoscope, the Roux limb is easily identified by only removing the greater omentum adhered to the porta hepatis. After partly dismantling the tail of the Roux limb, the fibrous occlusive hilar plate could be spotted. At this point, the main portal vein and its branches should be clearly and accurately marked, and the resection of the fibrous plate should be confined to the area between the portal branches. The resection level of the fibrous plate depends on the bile drainage status, which is consistent with that in ILKPE. The last step of portoenterostomy in RLKPE is the same as that in ILKPE [15-18].

However, some authors were reluctant to repeat the Kasai operation because of the possibility of a high incidence of perioperative complications (e.g., uncontrolled intraoperative bleeding) and longer ORT [13]. Perineal adhesion was more common in patients who underwent RLKPE. Specifically, in the vicinity of the porta hepatis, dense fibrous granulation tissue sometimes may result in unanticipated bleeding and damage to the Roux limb [11]. In some cases, oozing bleeding from the remains of the hilar fibrous plate is difficult to stop under laparoscopy. In addition, accidental portal hemorrhage from iatrogenic injuries may place patients at great risk. In our study, however, fewer peritoneal adhesions around the porta hepatis were observed during RLKPE, at least partially due to the laparoscopic technique used for ILKPE. Under laparoscopy, the image could be zoomed in, and the portal vein could be easily recognized, which may help to protect it from accidental injuries when dissecting the hilar fibrous plate [19]. Active bleeding at the fibrous stump can be stopped by compression with a gauze pad for a period. In the event of uncontrolled hemorrhage, conversion to OKPE should be adopted without taking risks to ensure the safety of the patients. The above measures adopted in our hospital may make RLKPE safer. In our study, the blood loss volume, conversion rate, ORT and incidence of APOC of RLKPE were not significantly different from those of ILKPE. Perhaps the technique of RLKPE may repeatedly be used for revision in patients with recurrent jaundice postoperatively. 
Two limitations of our study deserve comment. First, the evaluation of RLKPE was based on retrospective data. Second, there were only a few cases of RLKPE compared with the number of unrevised ILKPE and failed ILKPE cases. Nonetheless, we recommend RLKPE as the preferred surgical treatment for patients with recurrent jaundice after the initial Kasai operation. If the time interval between the cessation of bile drainage and revision is shortened, the postoperative outcomes could be improved.

\section{Conclusions}

In conclusion, RLKPE is a feasible, safe and effective procedure for the treatment of patients with recurrent jaundice after ILKPE. However, studies with a larger number of patients and a longer follow-up time are needed to confirm the advantages of this technique.

\section{Abbreviations}

BA: biliary atresia; RLKPE:revision of laparoscopic Kasai portoenterostomy; ILKPE:initially successful laparoscopic Kasai portoenterostomy; SNL:survival with native liver; LT:liver transplantation; OKPE:open Kasai portoenterostomy; $\mathrm{CJ}$ :clearance of jaundice; ORT:operative time; APOC:any perioperative complications

\section{Declarations}

\section{Ethical approval and consent to participate}

The study was approved by the Ethics Committee of the West China Hospital of Sichuan University. Written informed consent was obtained from the patients' parents, according to the provisions of the Declaration of Helsinki.

\section{Consent to publish}

Written informed consent for publication this study was obtained from the patients' parents. Copies of the signed informed consent forms are available for review by the Series Editor of BMC Pediatrics.

\section{Availability of data and materials}

The datasets used and/or analyzed during the current study available from the corresponding author on reasonable request.

\section{Competing interests}

The authors declare that they have no competing interests, either financial or non-financial, that could be perceived as prejudicing the impartiality of the research reported. 


\section{Funding}

This work was supported by the National Natural Science Foundation of China (grant numbers 81400862 and 81401606), the Key Project in the Science \& Technology Program of Sichuan Province (grant number 2019YFS0322), the Science Foundation for The Excellent Youth Scholars of Sichuan University (grant number 2015SU04A15), and the 1.3.5 Project for Disciplines of Excellence-Clinical Research Incubation Project, West China Hospital of Sichuan University (grant numbers 2019HXFH056 and 2020HXFH048).

\section{Funding}

This work was supported by grants from the National Natural Science Foundation of China (Grant No: 81401606 and 81400862), the Key Project in the Science \& Technology Program of Sichuan Province (Grant No: 2019YFS0322), the Science Foundation for Excellent Youth Scholars of Sichuan University (Grant No: 2015SU04A15), and the 1.3.5 Project for Disciplines of Excellence-Clinical Research Incubation Project, West China Hospital of Sichuan University (Grant No: 2019HXFH056 and 2020HXFH048).

\section{Authors' contributions}

$Y J, X P Z, S Y C, Y N L, K Y Y, J Y Z$, and ZCX were involved in the clinical management of these patients and collected clinical details of this study. ZCX reviewed the literature, and drafted the manuscript. YJ reviewed the manuscript. All authors read and approved the final manuscript.

\section{Acknowledgement}

We thank the patients and their parents for their co-operation and support, and for providing consent for publication. We also thank the study and hospital nurses for their assistance with the management of the patients.

\section{References}

1. Hartley JL, Davenport M, Kelly DA. Biliary atresia. Lancet. 2009;374:1704-13.

2. Lucianetti A, Guizzetti M, Bertani A, Corno V, Maldini G, Pinelli D, Aluffi A, Codazzi D, Spotti A, Spada M, Gridelli B, Torre G, Colledan M. Liver transplantation in children weighting less than $6 \mathrm{~kg}$ : the Bergamo experience. Transplant Proc. 2005;37:1143-5.

3. Hsu EK, Shaffer ML, Gao L, Sonnenday C, Volk ML, Bucuvalas J, Lai JC. Analysis of Liver Offers to Pediatric Candidates on the Transplant Wait List. Gastroenterology. 2017;153:988-95.

4. Esteves E, Clemente Neto E, Ottaiano Neto M, Devanir J Jr, Pereira E R. Laparoscopic Kasai portoenterostomy for biliary atresia. Pediatr Surg Int. 2002;18:737-40. 
5. Ji Y, Yang K, Zhang X, Jin S, Jiang X, Chen S, Xu Z. (2020) The short-term outcome of modified laparoscopic Kasai portoenterostomy for biliary atresia. Surg Endosc.

6. Ji Y, Yang K, Zhang X, Chen S, Xu Z. Learning curve of laparoscopic Kasai portoenterostomy for biliary atresia: report of 100 cases. BMC Surg. 2018;18:107.

7. Li Y, Xiang B, Wu Y, Wang C, Wang Q, Zhao Y, Chen S, Ji Y, Xu Z. Medium-term Outcome of Laparoscopic Kasai Portoenterostomy for Biliary Atresia With 49 Cases. J Pediatr Gastroenterol Nutr. 2018;66:857-60.

8. Wada M, Nakamura H, Koga H, Miyano G, Lane GJ, Okazaki T, Urao M, Murakami H, Kasahara M, Sakamoto S, Ishizaki Y, Kawasaki S, Yamataka A. Experience of treating biliary atresia with three types of portoenterostomy at a single institution: extended, modified Kasai, and laparoscopic modified Kasai. Pediatr Surg Int. 2014;30:863-70.

9. Yamataka A, Lane GJ, Koga H, Cazares J, Nakamura H. Role of laparoscopy during surgery at the porta hepatis. S Afr Med J. 2014;104:820-4.

10. Nio M, Sasaki H, Tanaka H, Okamura A. Redo surgery for biliary atresia. Pediatr Surg Int. 2013;29:989-93.

11. Mendoza MM, Chiang JH, Lee SY, Kao CY, Chuang JH, Tiao MM, Hsieh CS. Reappraise the effect of redo-Kasai for recurrent jaundice following Kasai operation for biliary atresia in the era of liver transplantation. Pediatr Surg Int. 2012;28:861-4.

12. Shirota C, Uchida H, Ono Y, Murase N, Tainaka T, Yokota K, Oshima K, Shirotsuki R, Hinoki A, Ando H. Long-term outcomes after revision of Kasai portoenterostomy for biliary atresia. J Hepatobiliary Pancreat Sci. 2016;23:715-20.

13. Murase N, Uchida H, Ono Y, Tainaka T, Yokota K, Tanano A, Shirota C, Shirotsuki R. (2015) A New Era of Laparoscopic Revision of Kasai Portoenterostomy for the Treatment of Biliary Atresia. Biomed Res Int 2015:173014.

14. Shneider BL, Brown MB, Haber B, Whitington PF, Schwarz K, Squires R, Bezerra J, Shepherd R, Rosenthal $\mathrm{P}$, Hoofnagle JH, Sokol RJ. A multicenter study of the outcome of biliary atresia in the United States, 1997 to 2000. J Pediatr. 2006;148:467-74.

15. Haber BA, Erlichman J, Thayu M, Flake AW, Rand EB. Successful revision of portoenterostomy in an infant with biliary atresia. J Pediatr Surg. 2006;41:e1-3.

16. Bondoc AJ, Taylor JA, Alonso MH, Nathan JD, Wang Y, Balistreri WF, Bezerra JA, Ryckman FC, Tiao $\mathrm{GM}$. The beneficial impact of revision of Kasai portoenterostomy for biliary atresia: an institutional study. Ann Surg. 2012;255:570-6.

17. Urahashi T, Ihara Y, Sanada Y, Wakiya T, Yamada N, Okada N, Mizuta K. Effect of repeat Kasai hepatic portoenterostomy on pediatric live-donor liver graft for biliary atresia. Exp Clin Transplant. 2013;11:259-63.

18. Sugawara Y, Makuuchi M, Kaneko J, Ohkubo T, Mizuta K, Kawarasaki H. Impact of previous multiple portoenterostomies on living donor liver transplantation for biliary atresia. Hepatogastroenterology. 2004;51:192-4. 
19. Li Y, Gan J, Wang C, Xu Z, Zhao Y, Ji Y. Comparison of laparoscopic portoenterostomy and open portoenterostomy for the treatment of biliary atresia. Surg Endosc. 2019;33:3143-52.

\section{Figures}

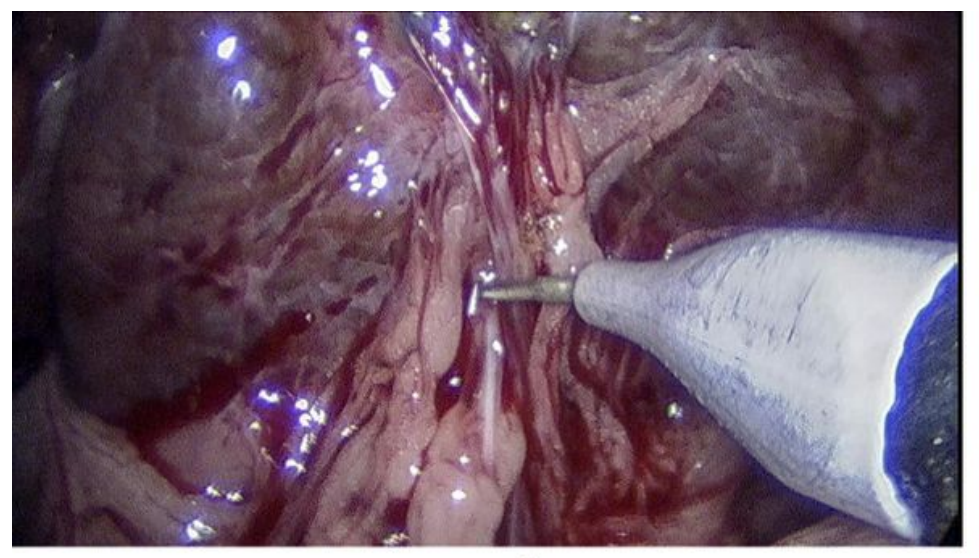

A

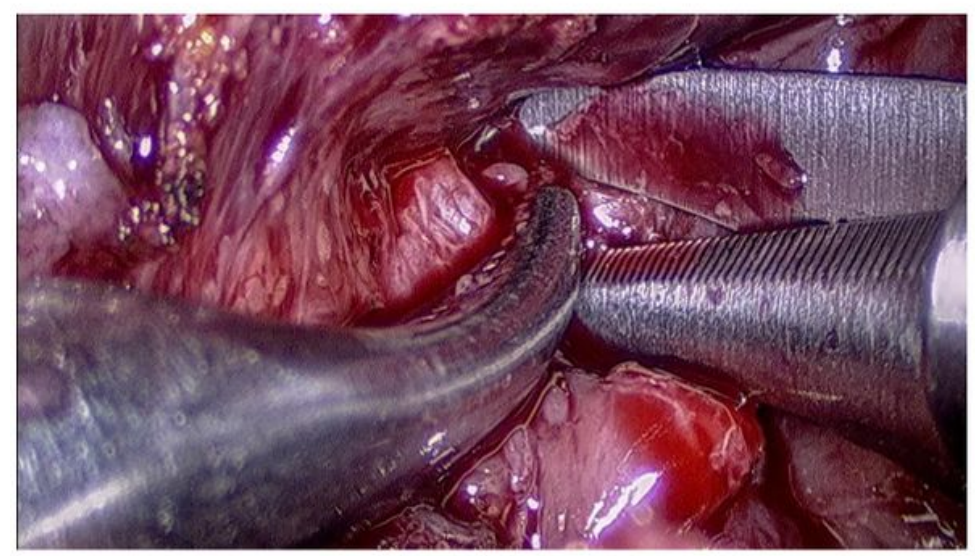

C

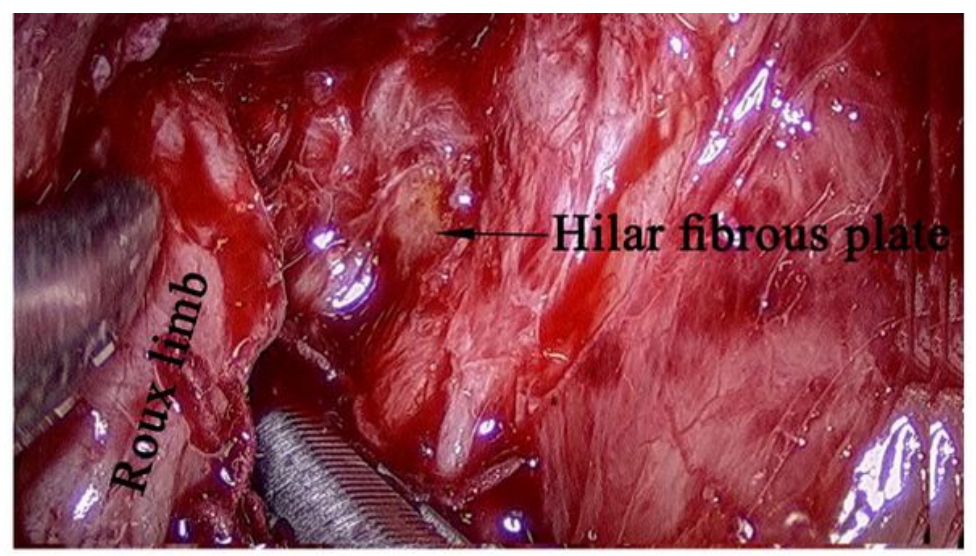

B

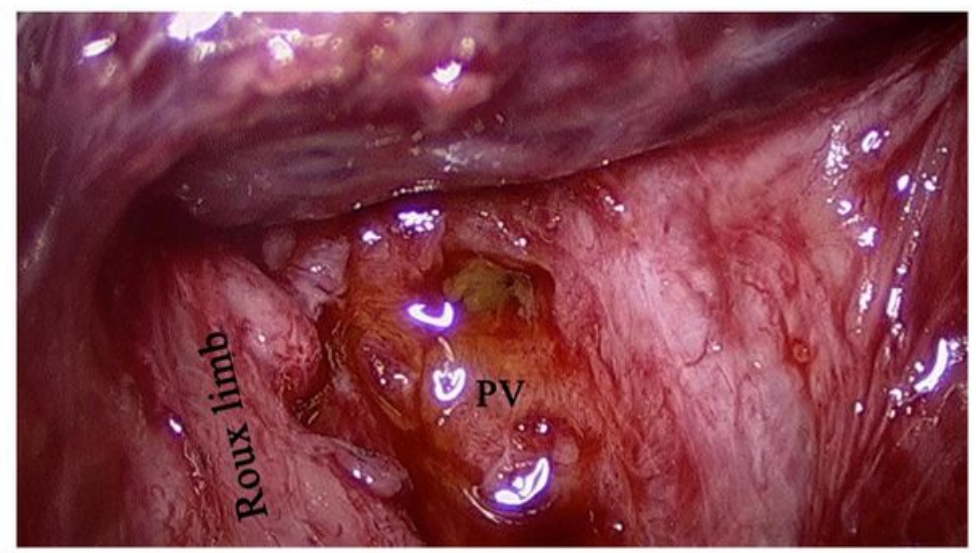

D

\section{Figure 1}

(a) During laparoscopic revision of Kasai portoenterostomy, few adhesions were found around the porta hepatis in patients who underwent initially successful laparoscopic Kasai portoenterostomy. (b) After dismantling the head of the Roux limb, the hilar fibrous plate could be identified. (c) The granulation and fibrous tissues were resected at the hilar plate before exposing the liver parenchyma. (d) The resection level of the fibrous cone depends on the presence of abundant bile-like juice over the fibrous stump. P V: portal vein. 


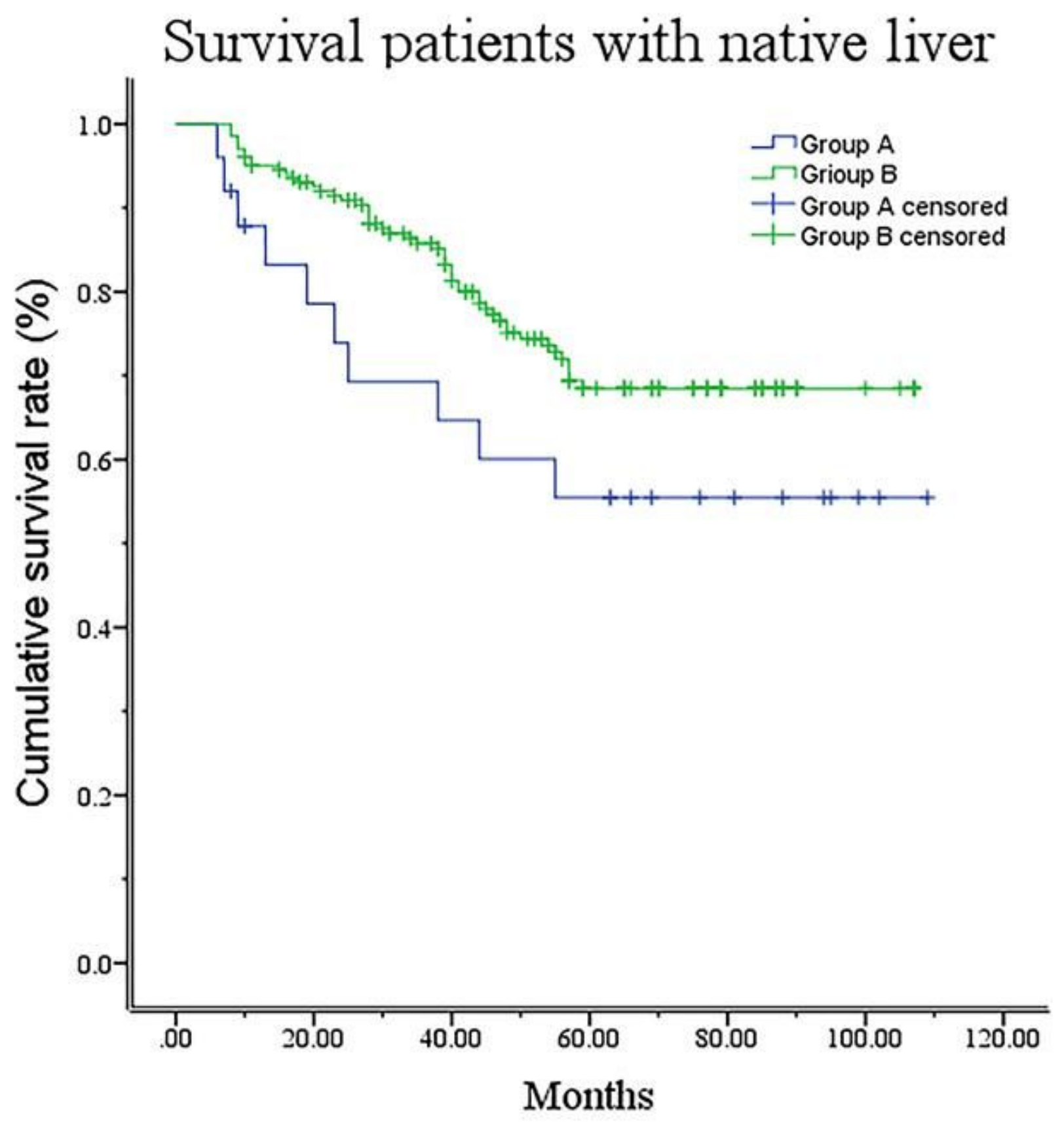

Figure 2

Kaplan-Meier analysis showed that the survival of patients with native livers was not significantly different between groups $A$ and $B(P>0.01)$. 


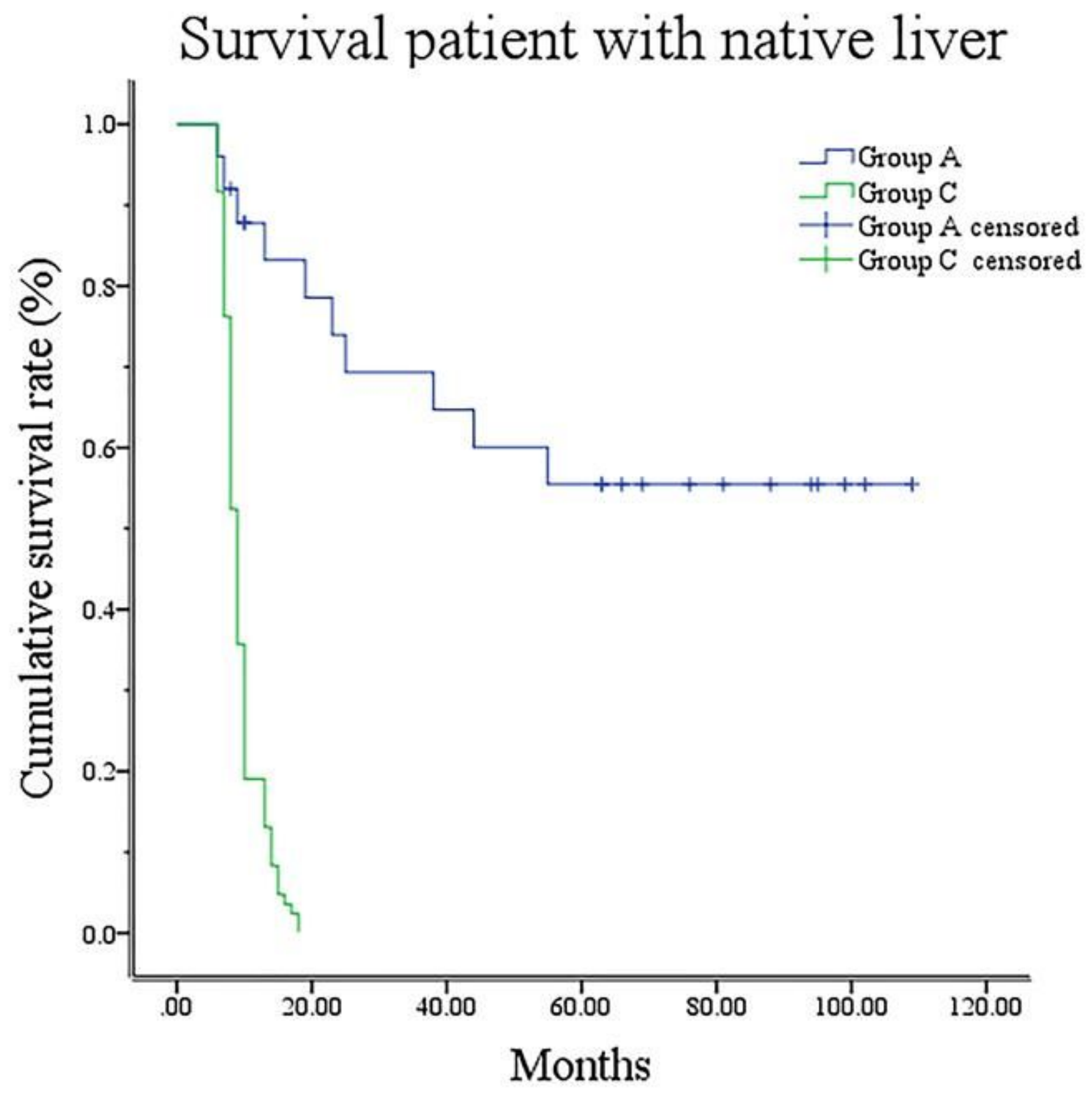

Figure 3

Kaplan-Meier analysis showed that the survival of patients with native livers was significantly different between groups $A$ and $C(P<0.01)$. 